EESTI NSV TEADUSTE AKADEEMIA TOIMETISED. XI KÖIDE FOOSIKALIS-MATEMAATILISTE JA TEHNILISTE TEADUSTE SEERIA. 1962, NR. 3

ИЗВЕСТИЯ АКАДЕМИИ НАУК ЭСТОНСКОИ ССР. ТОМ ХІ СЕРИЯ ФИЗИКО-МАТЕМАТИЧЕСКИХ И ТЕХНИЧЕСКИХ НАУК. 1962, № 3

\title{
СОПРОТИВЛЕНИЕ ПЕЧАТНОЙ ОБМОТКИ НА ДИСКООБРАЗНОМ РОТОРЕ
}

\section{В. ТАЕЛ}

\section{А. Введение}

Одним из факторов, от которого зависит величина потерь в машине (и тем самым к.п.д. машины), а также электромагнитная постоянная времени цепи якоря, является сопротивление обмотки якоря. Ниже предлагается методика и выводятся формулы для расчета сопротивления печатной обмотки дискового якоря.

Сопротивление якорной обмотки можно условно рассматривать как сумму из трех составляющих:

$$
\mathrm{R}=\mathrm{R}_{\mathrm{a}}+\mathrm{R}_{\mathrm{n}}+\mathrm{R}_{\mathrm{n}}
$$

где $\mathbf{R}_{\mathrm{a}}$ - сопротивление активных проводников;

$\mathbf{R}_{л}$ - сопротивление проводников лобовых соединений;

$\mathbf{R}_{\mathbf{n}}$ - сопротивление переходных через диск частей лобовых соединений.

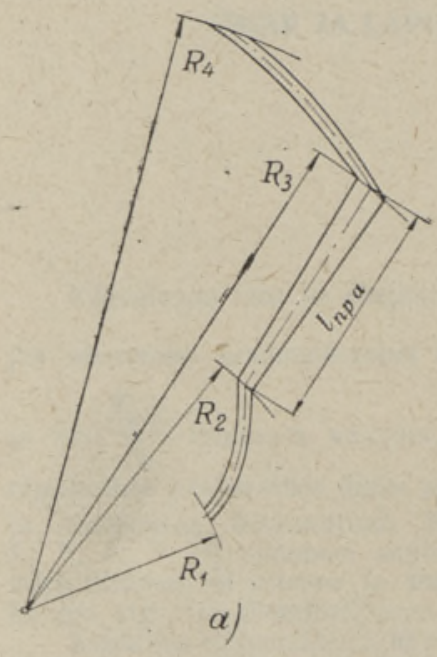

Фиг. 1

Активными проводниками называются те части, обмотки, которые размещаются между радиусами $R_{2}$ и $R_{3}$ (рис. 1). Проводники лобовых соединений образуются из частей, расположенных в областях между радиусами $R_{1}$ и $R_{2}$, а также $R_{3}$ и $R_{4}$. Все остальные части проводников, находящиеся вне области между $R_{1}$ и $R_{4}$, а также проходящие через диэлектрическую основу, образуют переходные части обмотки.

Для точного расчета сопротивления переходных частей $\mathbf{R}_{\text {п }}$ необходимо- знать подробно их состав и свойства, зависящие от технологии изготовления. Но их значение в общем сопротивлении роторной цепи невелико, и в приближенном расчете можно его и не учитывать. 
В статье выводятся формулы для сопротивления проводниковой части обмотки, т. е. для активных проводников и проводников лобовых соєдинений при постоянной по объему материала температуре и при оптимальном соотношении радиусов и оптимальной форме лобовых соединений (определенных в [ $\left.\left.{ }^{1}\right]\right)$. Предполагается, что слой материала проводников на диске имеет постоянную толщину $h=$ const.

При выводе сопротивления заменяем реальный проводник (рис. 1a) эквивалентным прямым проводником (рис. 1б), имеющим равную длину с осевой линией реального проводника во всех его частях.

\section{Б. Сопротивление проводника лобовой части}

Для лобовых частей, где ширина проводников $b=\mathrm{const}$, coпротивление проводника определено формулой

$$
\mathbf{R}_{\mathrm{np} \pi}=\frac{\varrho}{h b}\left[\int_{R_{1}}^{R_{2}} \frac{d R}{\cos a}+\int_{R_{3}}^{R_{4}} \frac{d R}{\cos a}\right]
$$

где $\mathbf{R}_{\text {прл }}$ - сопротивление проводника лобовых частей;

Q - удельное сопротивление материала;

$h$ - толщина проводника;

$b$ - ширина проводника;

$\alpha-$ угол между проводником и радиус-вектором, а $\cos \alpha=\frac{\boldsymbol{R}_{0}}{R}\left[{ }^{1}\right]$.

Зная, что ширина проводника лобовых соединений

$$
b=\lambda_{1} R_{0} \frac{4 \pi}{N}
$$

где $\lambda_{1}=1-\frac{b_{\text {на }} N}{4 \pi R_{0}} \quad$ коэффициент заполнения материалом проводника лобовых соединений;

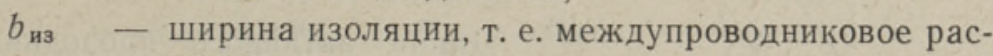
стояние;

$N \quad$ - число проводников на диске;

$R_{0} \quad$ - минимальный радиус обмотки,

и используя относительные величины радиусов

$$
r=\frac{R}{R_{3}}
$$

получим, проинтегрируя (2), выражение для сопротивления одного проводника лобовых соединений

$$
\mathbf{R}_{\mathrm{np} \Omega}=\frac{\varrho\left(r_{4}^{2}-r_{1}^{2}-\frac{2}{3}\right)}{8 \pi h r_{0}^{2}} \cdot \frac{N}{\lambda_{1}} .
$$




\section{В. Сопротивление активного проводника}

Сопротивление активного проводника в случае, когда ось активного проводника прямая и ширина изоляционного промежутка постоянная $b_{\text {вз }}=$ const, определена выражением

$$
\mathbf{R}_{\mathrm{npa}}=\frac{\varrho}{h} \int_{0}^{l_{\mathrm{npa}}} \frac{d l}{b_{a}}
$$

где $\mathbf{R}_{\text {пра }}$ - сопротивление активного проводника;

$t_{\text {nра }}$ - длина активного проводника (рис. 1);

$b_{a}$ - ширина активного проводника.

Ширина активного проводника в данном случае определена выражением

$$
b_{a}=\frac{\lambda_{3} R_{3} \frac{4 \pi}{N} \cos a_{a 3}-\lambda_{2} R_{2} \frac{4 \pi}{N} \cos a_{a 2}}{l_{\text {npa }}} l+\lambda_{2} R_{2} \frac{4 \pi}{N} \cos \alpha_{a 2},
$$

где $\quad \lambda_{3}-$ коэффициент заполнения материалом у радиуса $R_{3}$;

$\lambda_{2}$ - коэффициент заполнения материалом у радиуса $R_{2}$;

$\alpha_{a 3}$ и $\alpha_{a 2}$ - угол наклона активного проводника относительно радиус-вектора соответственно при радиусах $R_{3}$ и $R_{2}$;

$l$ - координат, направленный по оси активного проводника (рис. 1б).

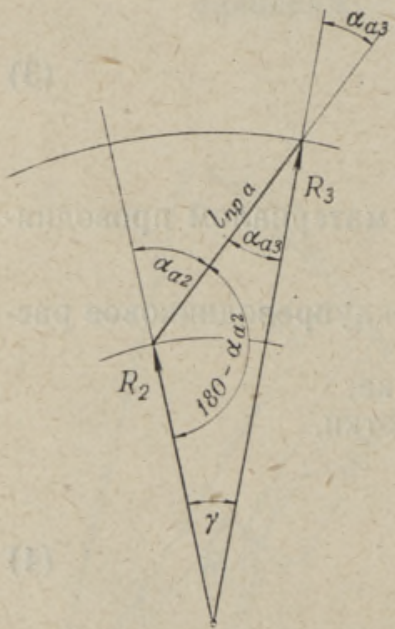

Фиг. 2.

Зная угол наклона активного проводника $\gamma$ (рис. 2), можно найти углы $\alpha_{a 2}$ и $\alpha_{a 3}$ непосредственно на чертеже или из соотношений

$$
\left.\begin{array}{r}
\operatorname{tg}\left(90^{\circ}+\frac{\gamma}{2}-\alpha_{a 2}\right)=\frac{1-r_{2}}{1+r_{2}} \operatorname{ctg}\left(\frac{\gamma}{2}\right) \\
\alpha_{a 3}=\alpha_{a 2}-\gamma .
\end{array}\right\}
$$

Длина активного проводника, в случае $\gamma \neq 0$, определена, с учетом, что $\sin \left(180^{\circ}-\right.$ $\left.-\alpha_{a 2}\right)=\sin \alpha_{a 2}$, выражением

$$
l_{\text {npa }}=\frac{R_{3} \sin \gamma}{\sin a_{a 2}} .
$$

Проинтегрируя теперь выражение (6), получим для сопротивления активного проводника

$$
\mathbf{R}_{\text {nрa }}=\frac{\varrho}{4 \pi h} \cdot \frac{R_{3} \sin \gamma}{\sin \alpha_{a 2}\left(\lambda_{3} R_{3} \cos \alpha_{a 3}-\lambda_{2} R_{9} \cos a_{a 2}\right)} \cdot \ln \left(\frac{\lambda_{3} R_{3} \cos \alpha_{a 3}}{\lambda_{2} R_{2} \cos a_{a 2}}\right) \cdot N .
$$

Учитывая, что $R_{2}=\frac{R_{3}}{\sqrt{3}}$, и выражая $\lambda_{2}$ и $\lambda_{3}$ из соотношений 


$$
\left.\begin{array}{l}
\lambda_{2}=1-\frac{b_{\text {из }} N}{4 \pi R_{2} \cos a_{a 2}} \\
\lambda_{3}=1-\frac{b_{\text {из }} N}{4 \pi R_{3} \cos a_{a 3}}
\end{array}\right\}
$$

получим для сопротивления активного проводника

$$
\mathbf{R}_{\text {пра }}=\frac{\varrho}{4 \pi h} \cdot \frac{\sin \gamma}{\sin a_{a 2}\left(\cos a_{a 3}-\frac{\cos a_{a 2}}{\sqrt{3}}\right)} \cdot \ln \left(\frac{\cos a_{a 3}-\frac{b_{\mathrm{и} 3} N}{4 \pi R_{3}}}{\frac{\cos a_{a 2}}{\sqrt{3}}-\frac{b_{\mathrm{и} 3} N}{4 \pi R_{3}}}\right) \cdot N
$$

С помощью несложной геометрической конструкции можно доказать, что

$$
\frac{\sin \gamma}{\sin a_{a 2}\left(\cos a_{a 3}-\frac{\cos a_{a 2}}{\sqrt{3}}\right)}=\mathrm{I}
$$

Итак, окончательно сопротивление одного прямого активного проводника, когда $b_{\text {из }}=$ const,

$$
\mathbf{R}_{\text {nра }}=\frac{\varrho}{4 \pi h} \cdot \ln \left(\frac{\cos a_{a 3}-\frac{b_{\text {из }} N}{4 \pi R_{3}}}{\frac{\cos a_{a 2}}{\sqrt{3}}-\frac{b_{\text {из }} N}{4 \pi R_{3}}}\right) \cdot N
$$

Полученная формула распространяется и на случай радиальных проводников, т. е. если $\gamma=0$. Здесь $\cos \alpha_{a 2}=\cos \alpha_{a 3}=1$.

\section{Г. Сопротивление обмотки}

На основе вышеизложенного, сопротивление якорной обмотки машины постоянного тока с печатной обмоткой на дискообразном якоре

$$
\begin{gathered}
\mathbf{R}=\frac{N}{4 a^{2}}\left(\mathbf{R}_{\text {пра }}+\mathbf{R}_{\text {прл }}\right)+\mathbf{R}_{\mathrm{n}}= \\
=\frac{\varrho N^{2}}{16 \pi a^{2} h}\left[\ln \frac{\cos a_{a 3}-\frac{b_{\text {из }} N}{4 \pi R_{3}}}{\frac{\cos a_{a 2}}{\sqrt{3}}-\frac{b_{\text {и }} N}{4 \pi R_{3}}}+\frac{r_{4}^{2}-r_{1}^{2}-\frac{2}{3}}{2 \lambda_{1} r_{0}^{2}}+\frac{16 \pi a^{2} h}{\varrho N^{2}} \mathbf{R}_{\mathrm{n}}\right],
\end{gathered}
$$

где $a-$ число параллельных ветвей.

Полученная формула выражает зависимость сопротивления обмотки от основных конструктивных факторов и дает возможность анализировать влияние последних на величину сопротивления.

Используя формулу (15) совместно с выражением тока [1]

$$
I=2 a \delta h r_{0} \frac{4 \pi}{N} \lambda_{i} R_{3}
$$


где $\delta$-- плотность тока,

можем написать формулу для потерь в якорной меди машины постоянного тока

$$
p_{\text {мя }}=I^{2} \mathrm{R}=4 \pi h \varrho \delta^{2} r_{0}^{2}\left[\ln \frac{\cos a_{a 3}-\frac{b_{\text {из }} N}{4 \pi R_{3}}}{\frac{\cos a_{a 2}}{\sqrt{3}}-\frac{b_{\text {из }} N}{4 \pi R_{3}}}+\frac{r_{4}^{2}-r_{1}^{2}-\frac{2}{3}}{2 \lambda_{1} r_{0}^{2}}+\frac{16 \pi a^{2} h}{\varrho N^{2}} \mathbf{R}_{\mathrm{n}}\right] \lambda_{1}^{2} R_{3}^{2} \text {. }
$$

Далее, положив в выражение электромагнитной мощности машины постоянного тока величину сопротивления $\mathrm{R}$ в виде (15), получим

$$
P^{\prime}=U I \mp I^{2}\left(\mathrm{R}+\frac{2 \Delta U}{I}\right)= \pm \frac{U E-E^{2}}{\mathbf{R}+\frac{2 \Delta U}{I}}
$$

где $\frac{2 \Delta U}{I}-$ сопротивление щеточных контактов,

т. е. имеем формулу, связывающую основные данные машины с ее другими электрическими и конструктивными параметрами.

Предшествующее рассмотрение основано на предположении, что температура материала обмотки по всему объему постоянная, что соответствует хंолодному состоянию машины. При работе машины разные части обмотки находятся в разных температурных условиях, которые зависят от распределения потерь и условий охлаждения.

Для точного учета влияния температуры на сопротивление необходимо определить ее распределение по всему объему обмотки при данном режиме работы. Предположим, что при установившемся режиме работы температура данной части обмотки зависит от ее расстояния от центра диска, тогда удельное сопротивление материала обмотки является функцией от радиуса $\mathrm{Q}=\mathrm{Q}(R)$ и должна оставаться в выражениях (2) и (6') под интегральным знаком.

В более простом случае можем влияние температуры учесть, вводя средние величины удельного сопротивления по частям обмотки. Так, если, например, на поверхности диска разделить три различающихся по температурным условиям зоны: 1) внутренние лобовые соединения, 2) активные части проводников и 3) внешние лобовые соединения, то выражение сопротивления обмотки (15) получится в виде

$$
\mathbf{R}=\frac{N^{2}}{16 \pi a^{2} h}\left[\varrho_{a} \ln \frac{\cos a_{a 3}-\frac{b_{\text {из }} N}{4 \pi R_{3}}}{\frac{\cos a_{a 2}}{\sqrt{3}}-\frac{b_{n 3} N}{4 \pi R_{3}}}+\frac{\varrho_{n 2}\left(r_{4}^{2}-1\right)+\varrho_{n 1}\left(\frac{1}{3}-r_{1}^{2}\right)}{2 \lambda_{1} r_{0}^{2}}+\frac{16 \pi a^{2} h}{N^{2}} \mathbf{R}_{\mathrm{n}}\right]
$$

где $\mathrm{л}$-. среднее удельное сопротивление материала внутренних лобовых соединений;

Qa - среднее удельное сопротивление материала активных проводников;

Qл2 - среднее удельное сопротивление материала внешних лобовых соединений. 


\title{
ЛИТЕ РА Т У РА
}

1. В. Т а л, О геометрии ротора с печатной обмоткой в электрических машинах, Изв. Акад. наук ЭССР, серия физ.-матем. и техн, наук, 1961, № 3.

\author{
Институт энергетики \\ Академии наук Эстонской ССР
}

Поступила в редакцию

26. X 1961

\section{TRUKITUD MÄHISE AKTIIVTAKISTUS KETTAKUJULISEL ROOTORIL}

\section{Tael \\ Resümee}

Artiklis tuletatakse kettakujulisele alusele trükitud mähise aktiivtakistuse avaldis. Aluseks on võetud konstantse vasekihi paksusega, sirgete aktiivjuhtmetega ja konstantse juhtmetevahelise isolatsioonivahemikuga mähis, ühtlase temperatuuriga kogu mähise ulatuses. Tuuakse avaldis alalisvoolumasina ankrumähise vaseskadudele. Masina töötemperatuurist tingituna on materjali eritakistus mähise ulatuses erinev, mida lihtsamal juhul vōib arvesse võtta keskmiste eritakistuste väärtustega lähedaste temperatuuritingimustega mähise osadele.

Eesti NSV Teaduste Akadeemia

Energeetika Instituut

Saabus toimetusse

26. X 1961

\section{ARMATURE RESISTANCE OF THE WINDING OF A PRINTED DISC ROTOR}

\section{Tael}

\section{Summary}

The author deduces an expression for the armature resistance of the printed winding of a disc rotor. A winding is taken which has a constant thickness of copper, straight active conductors with a constant insulation interval between the conductors, and a uniform temperature throughout the rotor. An expression for the ohmic losses in the armature of a d. c. machine is deduced. Owing to the working temperature of the machine, the specific resistance of the material within the range of the disc is different and can, in the simplest cases, be taken into consideration on the basis of the mean specific values for the parts of the disc having approximately equal temperature conditions.

Academy of Sciences of the Estonian S. S. R., Received Institute of Energetics 